Article

\title{
Hydrothermal Carbonation Carbon-Coated CdS Nanocomposite with Enhanced Photocatalytic Activity and Stability
}

\author{
Yuanliang Ma ${ }^{1}$, Zhongkun Zhao ${ }^{2}$, Zhurui Shen ${ }^{2, *}$, Qiang Cai ${ }^{2, *}$, Huiming $\mathrm{Ji}^{2}$ and \\ Leichao Meng ${ }^{1}$ \\ 1 College of Physics and Electronic Information Engineering, Qinghai University for Nationalities, \\ Xining 810007, China; mayuanliang@163.com (Y.M.); mengleichao@163.com (L.M.) \\ 2 Key Laboratory of Advanced Ceramics and Machining Technology, Ministry of Education, School of \\ Materials Science and Engineering, Tianjin University, Tianjin 300072, China; 18222490722@163.com (Z.Z.); \\ jihuiming@tju.edu.cn (H.J.) \\ * Correspondence: shenzhurui@tju.edu.cn (Z.S.); caiqtju@163.com (Q.C.); Tel.: +86-02227890485 (Z.S. \& Q.C.)
}

Academic Editors: Tianyi Ma, Jian-Rong (Jeff) Li and Cláudia Gomes Silva

Received: 3 May 2017; Accepted: 19 June 2017; Published: 24 June 2017

\begin{abstract}
Herein, a novel CdS nanocomposite is fabricated by a facile one-pot hydrothermal method assisted by glucose and polyvinylpyrrolidone (PVP). The as-prepared CdS is coated with a thin layer, which is determined to be hydrothermal carbonation carbon (HTCC) mainly containing semiconductive polyfuran. The as-prepared HTCC-coated CdS shows superior photocatalytic activity for the degradation of Rhodamine B (RhB) under visible light irradiation $(\lambda \geq 420 \mathrm{~nm})$. The optimum sample (glucose content of $0.1 \mathrm{~g}$ ) shows a degradation rate four-times that of pure CdS reference. Moreover, it also shows an improved stability, and the activity can be maintained at $96.2 \%$ after three cycles of recycling. The enhanced photocatalytic activity and stability of nanocomposite can mainly be attributed to: (i) The addition of PVP in the reaction solution can significantly increase the specific surface area of CdS and thus offer more active sites. (ii) The HTCC in the nanocomposite can expand the range of light absorption. (iii) The HTCC layer can form a heterojunction with CdS and improve the charge separation and transfer.
\end{abstract}

Keywords: CdS; hydrothermal carbonation carbon; nanocomposite; photocatalysis

\section{Introduction}

Environmental pollution and the energy crisis have become two of the most severe worldwide problems in the 21st century. Photocatalysis is a very promising technique for both energy and environmental issues due to its direct utilization of solar energy [1,2]. In the past four decades, various semiconductors had been developed to be used as photocatalyst, such as $\mathrm{TiO}_{2}$ [3], graphite carbon nitride [4], $\mathrm{BiVO}_{4}$ [5], and other newly emerging materials [6]. However, to date, it is still a great challenge to develop an applicable photocatalyst.

Among various photocatalysts, cadmium sulfide (CdS) is a well-known visible-light-driven material. As a widely-used semiconductor, it has a direct bandgap of around $2.4 \mathrm{eV}$, which matches well with visible light spectrum, and exhibits excellent photocatalytic activity under sunlight or visible light irradiation $[7,8]$. Thus, it has been applied in a wide range of fields, including optoelectronics, photovoltaics, and photocatalysis [9]. However, the CdS-based photocatalyst can hardly show its full potential in photocatalysis because of its high recombination rate of photoexcited electron-hole pairs and inherent photocorrosion problem under illumination [10]. Moreover, the CdS nanoparticles generally aggregate, thus leading to low specific surface area and absorption ability [11]. Generally, there are three 
ways to overcome these problems and develop high-efficiency CdS-based photocatalyst. One is the loading of co-catalysts $[12,13]$ to enhance the charge separation and transfer. The second is coupling with nanomaterials such as graphene oxide (GO) [14] and carbon nanotubes (CNTs) [15] which have large specific surface area in order to increase the active sites. The third strategy is to coat CdS with a thin layer of carbon-based materials (e.g., amorphous carbon [16] or fullerene [17]), which is an effective method to enhance the charge separation and protect the surface from photocorrosion. Hydrothermal carbonation carbon (HTCC) is a kind of highly functionalized carbon material [18] which can couple with metal oxide photocatalysts such as $\mathrm{MoO}_{2}$ and $\mathrm{WO}_{3}$ and establish a heterojunction to enhance the charge separation [19]. However, to the best of our knowledge, there are few reports of HTCC-enhanced CdS.

Herein, we report a facile one-pot hydrothermal preparation $\left(180^{\circ} \mathrm{C}\right.$ for $\left.10 \mathrm{~h}\right)$ of HTCC-coated CdS nanocomposite assisted by glucose and polyvinylpyrrolidone (PVP). The PVP is used to decrease the agglomeration of CdS nanoparticles in the reaction system, and the glucose works as the carbon source. The amorphous coating layer of CdS is determined to be HTCC, which mainly contains semiconductive polyfuran. This HTCC-coated CdS shows superior photocatalytic activity and stability for the degradation of RhB under visible light irradiation $(\lambda \geq 420 \mathrm{~nm})$. This enhancement in activity and stability can mainly be attributed to: (i) The addition of PVP in the reaction solution can significantly increase the specific surface area of $\mathrm{CdS}$, and thus offer more active sites; (ii) The HTCC in the nanocomposite can expand the range of light absorption; (iii) The HTCC layer can form a heterojunction with CdS and improve the charge separation and transfer. This novel CdS-based nanocomposite exhibits great potential in environmental remediation.

\section{Results and Discussion}

$\mathrm{X}$-ray diffraction $(\mathrm{XRD})$ analysis was used to investigate the crystallographic phase of pure CdS and $0.1 \mathrm{C} / \mathrm{CdS}$ samples. As shown in Figure 1, all of the diffraction peaks match well to the hexagonal phase of CdS (JCPDS card No. 41-1049). The peaks at $24.8^{\circ}, 26.5^{\circ}, 28.2^{\circ}, 36.6^{\circ}, 43.7^{\circ}, 47.8^{\circ}, 51.8^{\circ}$, and $52.8^{\circ}$ correspond to (100), (002), (101), (102), (110), (103), (112), and (201) planes of hexagonal CdS, respectively. There is no obvious difference of XRD pattern between pure CdS and 0.1C/CdS sample, implying that the crystalline structure of CdS remains unchanged after the introduction of glucose and PVP.

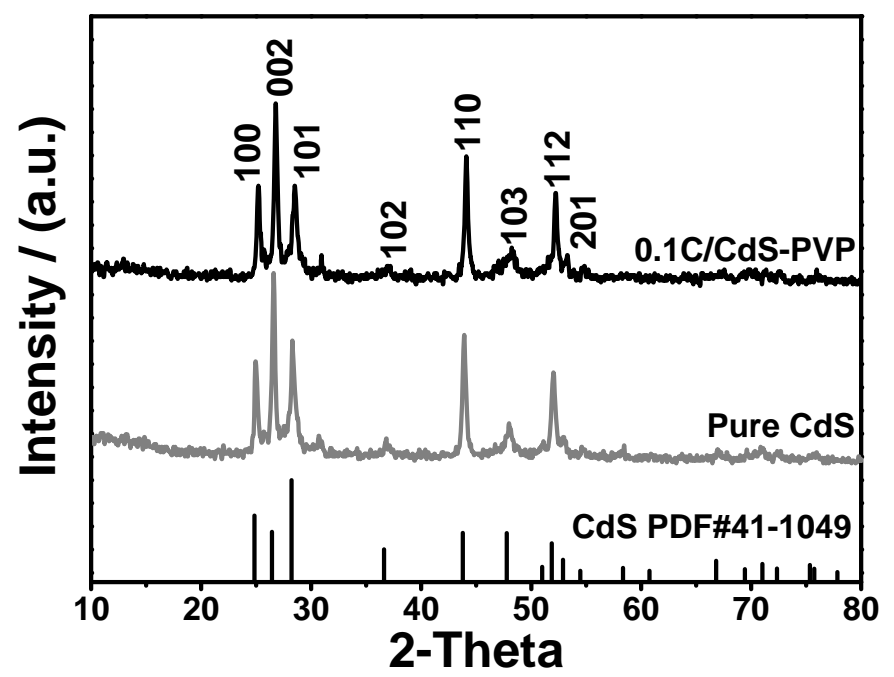

Figure 1. XRD patterns of the as-prepared pure CdS and 0.1C/CdS-PVP samples. PVP: polyvinylpyrrolidone.

Fourier transform infrared (FT-IR) spectroscopy was conducted to investigate the chemical structure of CdS-PVP, 0.1C/CdS-PVP, and HTCC (Figure 2). For CdS-PVP and 0.1C/CdS-PVP samples, the weak band around $565 \mathrm{~cm}^{-1}$ indicates $-\mathrm{CS}_{2}$ wagging (Cd-S stretching) [20]. The bands at 1375 and $1450 \mathrm{~cm}^{-1}$ 
can be attributed to the Cd-S bonding [21]. Moreover, both samples contain some characteristic peaks of PVP, such as $1283 \mathrm{~cm}^{-1}, 1414 \mathrm{~cm}^{-1}$, and $1658 \mathrm{~cm}^{-1}$. For the HTCC, the peaks at $1605 \mathrm{~cm}^{-1}, 1448 \mathrm{~cm}^{-1}$, $1365 \mathrm{~cm}^{-1}$, and $961 \mathrm{~cm}^{-1}$ can be assigned to the vibrational modes of the furan monomer [22]. The band at $1509 \mathrm{~cm}^{-1}$ is ascribed to the $\mathrm{C}=\mathrm{C}$ stretching of furan ring in the polymer. Besides, the band at 794 $\mathrm{cm}^{-1}$ is attributed to the linear structure in polyfuran. Moreover, the bands at $1200 \mathrm{~cm}^{-1}$ and $1020 \mathrm{~cm}^{-1}$ are ascribed to the $\mathrm{C}-\mathrm{H}$ bending and stretching modes, and the band at $861 \mathrm{~cm}^{-1}$ is assigned to the out-of-plane bending of $\mathrm{C}-\mathrm{H}$ modes [22]. These bands come from the $\mathrm{C}-\mathrm{H}$ groups in the furan rings. The band $1702 \mathrm{~cm}^{-1}$ is ascribed to the $\mathrm{C}=\mathrm{O}$ stretching, suggesting that some furan rings are open in the polymer, which is common for the polyfuran. Moreover, ${ }^{13} \mathrm{C}$ solid-state CP-MAS NMR spectroscopy was also performed (Figure S1), and further confirmed the existence of polyfuran in the HTCC [19]. Therefore, we can infer that the HTCC is mainly composed of polyfuran components, which is in good agreement with previous studies [18,19]. Comparing the FT-IR spectra of HTCC and 0.1C/CdS-PVP, we can find some characteristic peaks of HTCC in the 0.1C/CdS-PVP sample, such as $861 \mathrm{~cm}^{-1}, 1020 \mathrm{~cm}^{-1}$, $1200 \mathrm{~cm}^{-1}$, and $1365 \mathrm{~cm}^{-1}$. Thus, we can conclude the existence of HTCC in the composite.

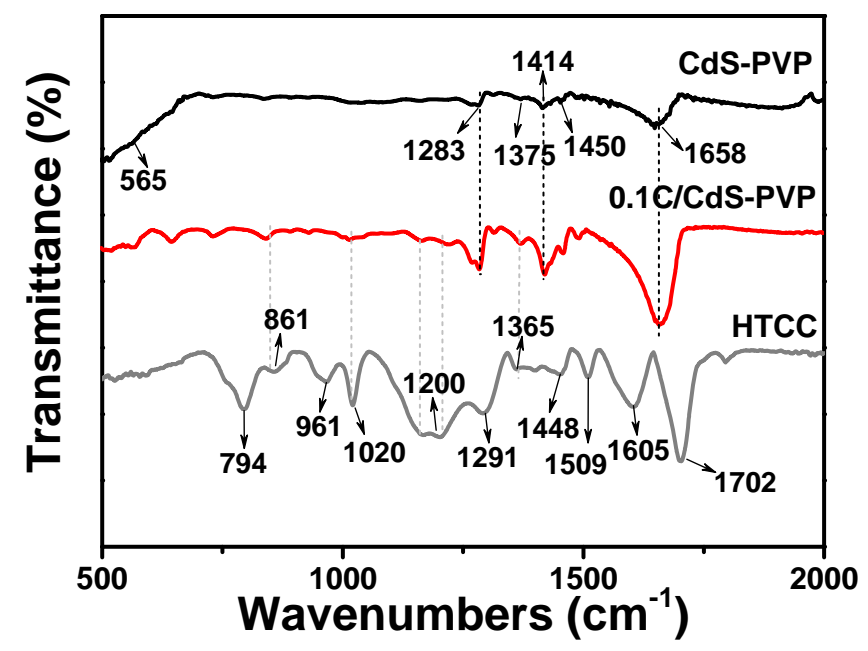

Figure 2. The FT-IR spectrum of as-prepared CdS-PVP, 0.1C/CdS-PVP, and HTCC.

The morphology and microstructure are investigated by scanning electron microscopy (SEM) and transmission electron microscopy (TEM) analysis in Figure 3. Figure 3a shows the TEM image of an individual pure CdS particle. As shown in Figure 3b, the 0.1C/CdS-PVP was mostly composed of nanocrystals with polygonal morphology with the size of c.a. $60 \mathrm{~nm}$, and others were irregular particles. Figure $3 c$ displays a TEM image of a 0.1C/CdS-PVP particle. In Figure $3 c$, a homogeneous amorphous layer coating on the surface of CdS nanocrystals with intimate contact can be clearly observed. This TEM image demonstrates that the 0.1C/CdS-PVP consisted of core-shell structures. Combining the results of XRD and FT-IR, we can infer that the amorphous layer is HTCC components in the 0.1C/CdS-PVP sample. Figure 3d displays a high-resolution TEM (HRTEM) image of 0.1C/CdS-PVP sample. The lattice fringes are measured to be $0.316 \mathrm{~nm}$, which agree well with the (111) plane of hexagonal CdS. Through HRTEM observation, it can be measured that the thickness of the HTCC layer was as thin as 4-6 nm, which will not block the visible light illumination to the CdS. As expected, the layer was observed in other CdS/C-PVP nanocomposites (Figure S2). The thickness of the HTCC layer varied from 2 to $10 \mathrm{~nm}$ with the addition of glucose $(0.05-0.5 \mathrm{~g})$. The formation of the carbon shell may be formed by the adsorption of fractional amorphous carbon generated from the hydrothermal carbonization process. In addition, the thermal gravimetric analysis (TGA) curves of CdS-PVP and 0.1C/CdS-PVP samples are recorded from room temperature to $550{ }^{\circ} \mathrm{C}$ in air with a heating rate of $10^{\circ} \mathrm{C} \cdot \mathrm{min}^{-1}$ (Figure S3). The two samples display a decrease in weight loss from $300-500{ }^{\circ} \mathrm{C}$, which can be attributed to the decomposition of PVP, the oxidation of CdS, and the removal of HTCC. Thus, 
we infer that the difference between CdS-PVP and 0.1C/CdS-PVP samples is the amount of HTCC in $0.1 \mathrm{C} / \mathrm{CdS}-\mathrm{PVP}$, which is approximately $1.96 \mathrm{wt} \%$.

Figure 4 shows the nitrogen adsorption-desorption isotherms and corresponding Brunauer-Emmett-Teller (BET) surface area of the pure CdS, CdS-PVP, and 0.1C/CdS-PVP samples. As shown in Figure 4, the BET surface areas of the three samples were as follows: $10.537 \mathrm{~m}^{2} / \mathrm{g}$ for pure CdS, $30.366 \mathrm{~m}^{2} / \mathrm{g}$ for CdS-PVP, and $23.630 \mathrm{~m}^{2} / \mathrm{g}$ for $0.1 \mathrm{C} / \mathrm{CdS}-\mathrm{PVP}$. The corresponding pore size distributions of the three samples ranged from about $2 \mathrm{~nm}$ to $50 \mathrm{~nm}$ and centered at ca. $20 \mathrm{~nm}$ (Figure S4). It should be noted that the surface area of CdS-PVP was two times higher than that of pure CdS, even with the addition of a small amount of PVP $(0.3 \mathrm{~g})$. This is because PVP is a widely-used water dispersible and structure-directing reagent $[23,24]$, which is beneficial to avoiding the agglomeration of CdS particles. A photocatalyst with larger specific surface is beneficial for its photocatalytic activity because of enhanced absorption ability. Compared to CdS-PVP, the BET surface area of $0.1 \mathrm{C} / \mathrm{CdS}$-PVP sample decreases slightly. This phenomenon is caused by the coating carbon layer, which may work as adhesive to connect the dispersive CdS particles.

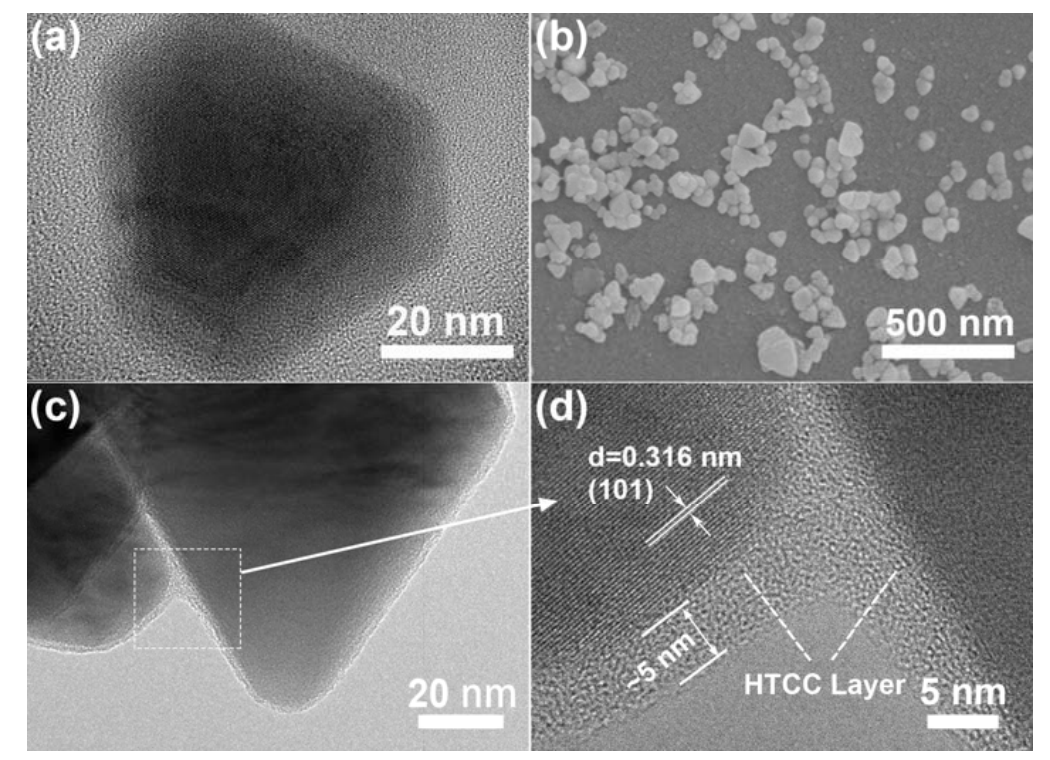

Figure 3. (a) TEM image of pure CdS sample; (b) SEM, (c) TEM, and (d) HRTEM images of $0.1 \mathrm{C} / \mathrm{CdS}-\mathrm{PVP}$ sample.

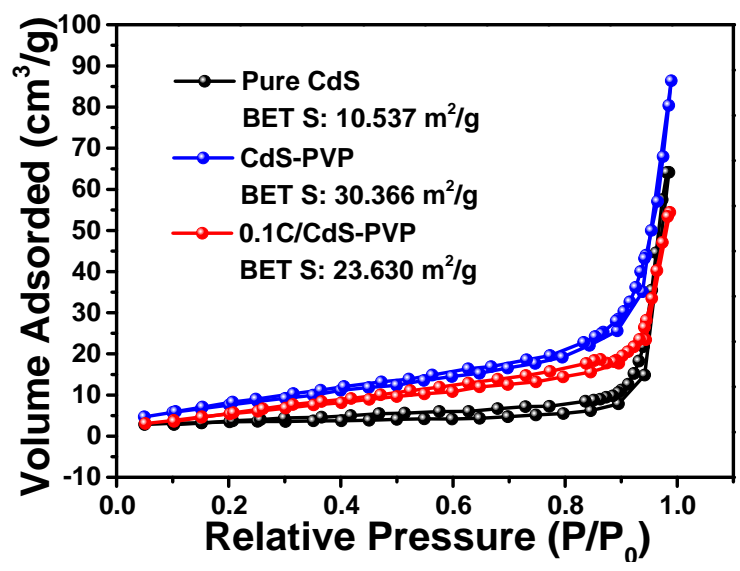

Figure 4. Nitrogen adsorption-desorption isotherms and corresponding Brunauer-Emmett-Teller (BET) surface area of the pure CdS, CdS-PVP, and 0.1C/CdS-PVP samples. 
A comparison of the UV-vis diffuse reflectance spectra of pure CdS, 0.05CdS-PVP, 0.1CdS-PVP, 0.2CdS-PVP, and 0.5CdS-PVP samples is displayed in Figure 5. All of the samples exhibit a similar absorption edge at around $520 \mathrm{~nm}$, which corresponds to the intrinsic band gap of CdS. Compared to pure CdS, the absorbance of visible light over all C/CdS-PVP samples was enhanced. Additionally, the color of CdS-PVP samples became darker with the increasing glucose contents. It is well-known that a darker photocatalyst will lead to an enhanced light absorption ability, thus improving its photocatalytic activity. In addition, the band gap of the samples is further determined by the linear approximation in the Kubelka-Munk plots (Figure S5). The results show that the band gaps of the C/CdS-PVP samples are similar.

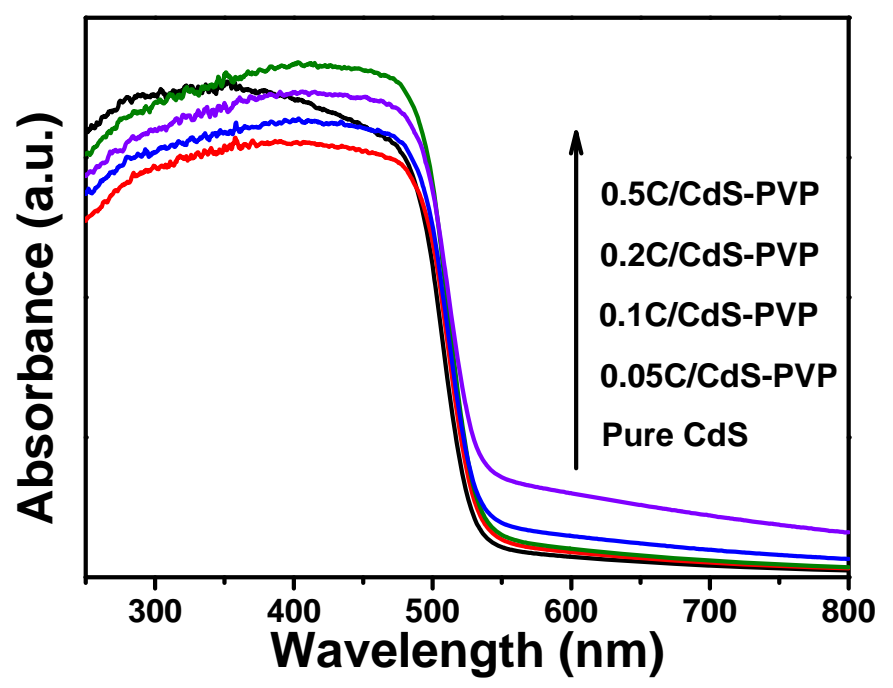

Figure 5. Comparison of UV-vis diffuse reflectance absorption spectra of the as-prepared samples.

To evaluate the photocatalytic performance of the as-prepared samples, photocatalytic degradation of $\mathrm{RhB}$ was conducted under visible light irradiation. Figure 6a presents the degradation efficiencies of C/CdS-PVP with different contents of glucose, pure CdS, and CdS-PVP samples. As presented in Figure 6a, pure CdS exhibits a limited degradation efficiency of $54.5 \%$ in 30 min due to the rapid recombination of charge carriers. However, the degradation efficiency of CdS-PVP increases to $80.0 \%$ with the introduction of PVP additive. Such a large increase benefits by the improved specific surface area and absorption ability of CdS-PVP. Additionally, the larger specific surface area of CdS-PVP has more active sites and reaction centers for photocatalytic reaction, which facilitate an enhanced activity. The degradation efficiency of C/CdS-PVP further increases after the carbon coating on the CdS. After irradiation for $30 \mathrm{~min}$, the degradation efficiencies over different C/CdS-PVP are 90.2\% (0.05C/CdS-PVP), 94.7\% (0.1C/CdS-PVP), 87.3\% (0.2C/CdS-PVP), and 49.0\% (0.5C/CdS-PVP). Therefore, we confirm that the optimum amount of glucose was $0.1 \mathrm{~g}$.

It can be observed that the 0.5C/CdS-PVP sample showed a very low activity, even lower than that of the pure CdS. This is attributed to two factors: (1) A greater content of glucose in the precursor will lead to thicker HTCC layer and decrease the light intensity on the surface of CdS. (2) The reaction rate of the carbonization of the glucose will become faster, resulting in the formation of carbon nanospheres with larger size when the concentration of glucose is raised (Figure S6). Thus, the large amounts of black carbon nanospheres would shield the visible light, which is called a "shielding effect" [14], resulting in a rapid decrease of activity. Therefore, a suitable content of glucose is crucial for the optimal activity. It should be pointed out that the $0.1 \mathrm{C} / \mathrm{CdS}$ sample also exhibits a low photocatalytic activity, similar to the pure CdS. This may be attributed to the serious agglomeration of CdS particles. In this case, there is only a small quantity of amorphous carbon that can adsorb on the surface of $\mathrm{CdS}$, and most tend to form carbon colloid spheres. Additionally, the corresponding reaction kinetics 
of the degradation are calculated and shown in Figure $6 \mathrm{~b}$. The 0.1C/CdS-PVP sample showed the highest apparent reaction rate, which is nearly four times as high as that of pure $\operatorname{CdS}\left(0.102 \mathrm{~min}^{-1} v\right.$. $\left.0.026 \mathrm{~min}^{-1}\right)$. Moreover, the $0.1 \mathrm{C} / \mathrm{CdS}-\mathrm{PVP}$ sample showed a superior stability compared to pure CdS. As shown in Figure $6 c$, there was only a 3.8\% decrease of degradation efficiency for $0.1 \mathrm{C} / \mathrm{CdS}-\mathrm{PVP}$ sample after three recycling degradation, while pure CdS had a $10.9 \%$ decrease.
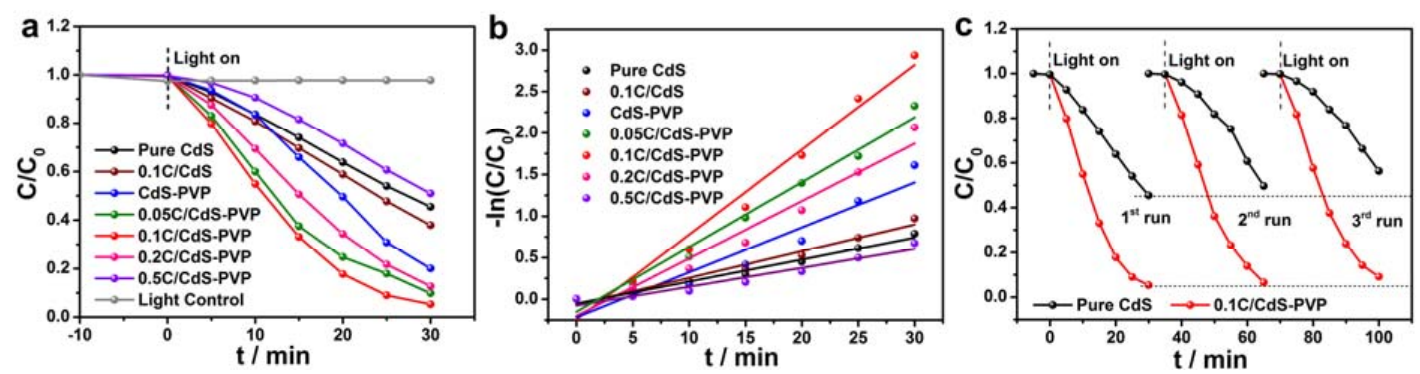

Figure 6. (a) Temporal course of the photodegradation of Rhodamine B (RhB) and (b) corresponding kinetic curves over different photocatalysts under visible light irradiation; (c) Recycled photodegradation of RhB under visible light irradiation over the pure CdS and 0.1C/CdS-PVP samples.

On the basis of the above results and discussion, we can infer that the PVP and the thin layer of HTCC have a significant effect on the process of photocatalytic degradation. Firstly, the addition of PVP in the reaction solution can significantly increase the specific surface area of CdS and offer more active adsorption sites and reaction centers. Secondly, the thin layer of HTCC can improve the adsorption ability of light. Finally, the separation of charge carriers in the CdS can be effectively improved.

Recently, our group found that the HTCC derived from glucose is a polyfuran-based $n$-type semiconductor, containing plenty of $\mathrm{sp}^{2}$-hybridization structures $[19,25]$. Under solar light illumination, HTCC generates photoexcited electrons, holes, and hydroxyl radicals. These species can be used for photocatalytic treatment such as water disinfection and the degradation of organic pollutants. Combining the FT-IR, ${ }^{13} \mathrm{C}$ NMR, XRD, and TEM, we can infer that the coverage layer on the surface of CdS is also HTCC mainly containing the polyfuran, like those in our previous report. Therefore, according to the above literatures, the conduction band (CB) and valence band (VB) position of HTCC are estimated to be about $-0.65 \mathrm{~V}$ (vs. NHE, Normal Hydrogen Electrode) and $+0.54 \mathrm{~V}$ (vs. NHE). Therefore, a proper p-n heterojunction is constructed base on the intimate contact between the CdS (the CB is $-0.97 \mathrm{~V}$ vs. NHE and VB is $+1.48 \mathrm{~V}$ vs. NHE) and HTCC (Figure 7a). Under illumination, the valence electrons $\left(\mathrm{e}^{-}\right)$in the CdS can be excited to the conduction band (CB) and leaving holes $\left(\mathrm{h}^{+}\right)$in the valence band (VB). Then, the holes in the CdS will transfer to the HTCC with higher valance band maximum and survive from being recombined. They can then migrate to the solution through the conductive polyfuran domains and participate in photocatalytic reactions. Therefore, only a fraction of holes can photocorrode the CdS nanocrystals, and achieve an enhanced photocatalytic activity and stability. On the other hand, the HTCC can also generate photoexcited electrons and transfer to the $\mathrm{CB}$ of $\mathrm{CdS}$, which is beneficial to the separation of photoinduced charge carriers. Moreover, the photoluminescence (PL) emission spectra were recorded to investigate the charge recombination process in pure CdS and 0.1C/CdS-PVP samples. As shown in Figure $7 \mathrm{~b}$, the pure CdS exhibited stronger PL intensity $0.1 \mathrm{C} / \mathrm{CdS}-\mathrm{PVP}$, suggesting the higher charge recombination process in the pure CdS. Consequently, a better photocatalytic performance of 0.1C/CdS-PVP is expected. 

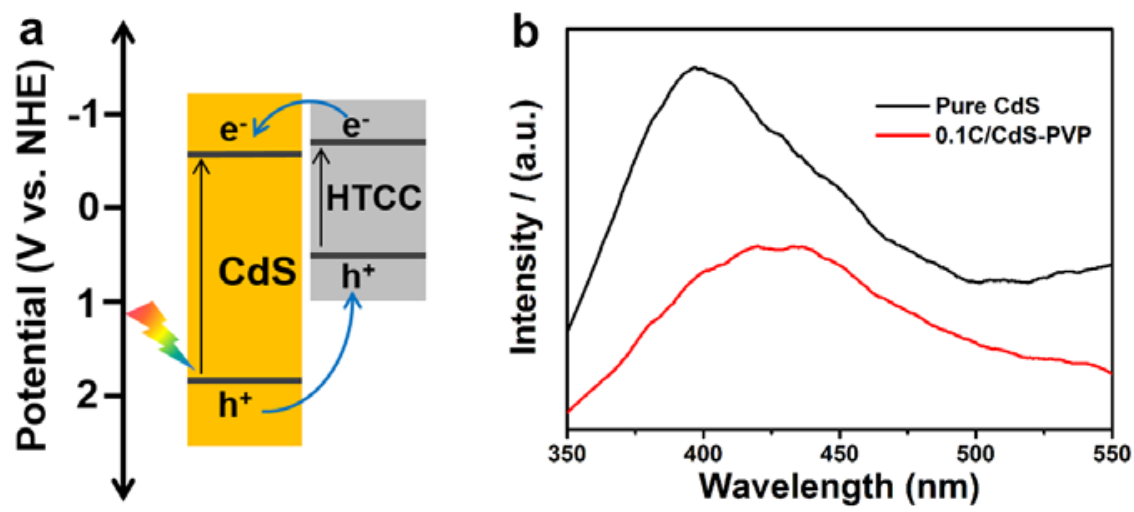

Figure 7. (a) Schematic illustration of the charge transfer process between CdS and Hydrothermal carbonation carbon (HTCC) in the HTCC-coated CdS nanocomposite under visible light irradiation; (b) Photoluminescence (PL) emission spectra of the pure CdS and 0.1C/CdS-PVP samples.

\section{Materials and Methods}

\subsection{Materials}

All reagents were used without any further purification. $\mathrm{Cd}\left(\mathrm{CH}_{3} \mathrm{COO}\right)_{2} \cdot 2 \mathrm{H}_{2} \mathrm{O}$ and L-cysteine were obtained from Aladdin Reagent Company (Shanghai, China). Polyvinylpyrrolidone (PVP; MW $\sim 58 \mathrm{~K}$ ) was purchased from Macklin (Shanghai, China). Glucose and Rhodamine B (RhB) were purchased from Yuanli Chemical Company (Tianjin, China). Deionized (DI) water used in all experiments was from local sources.

\subsection{Synthesis of Carbon-Coated CdS Nanocomposites}

The carbon-coated CdS nanocomposites were synthesized by a simple hydrothermal reaction. The PVP and glucose were used as dispersant and carbon source, respectively. The content of PVP was fixed. In a typical synthesis, $\mathrm{Cd}\left(\mathrm{CH}_{3} \mathrm{COO}\right)_{2} \cdot 2 \mathrm{H}_{2} \mathrm{O}(5 \mathrm{mmol})$, L-cysteine $(10 \mathrm{mmol})$, and PVP $(0.3 \mathrm{~g})$ were dissolved in $20 \mathrm{~mL}$ of DI water to form a homogeneous suspension by vigorous stirring for $1 \mathrm{~h}$. Then, glucose $(0.05-0.5 \mathrm{~g})$ was added into the suspension and kept stirring for another $1 \mathrm{~h}$ period. Subsequently, the suspension was transferred to a $30 \mathrm{~mL}$ Teflon-lined stainless steel autoclave and kept at $180^{\circ} \mathrm{C}$ for $10 \mathrm{~h}$. After the reaction, the autoclave was cooled to room temperature, and then the precipitates were collected by centrifugation and washed with DI water and ethanol before drying in a vacuum oven at $70{ }^{\circ} \mathrm{C}$ overnight. As a control, pure CdS and CdS-PVP were also synthesized by the same method with and without adding PVP. The as-prepared CdS samples were named as pure CdS, CdS-PVP, $x$ C/CdS, and $x$ C/CdS-PVP (where $x$ is the amount of glucose and $x=0,0.05,0.1,0.2,0.5$ ).

\subsection{Characterization}

The X-ray diffraction (XRD) patterns were obtained using a D8 Advanced X-ray diffractometer (Brooklyn, Germany). Scanning electron microscopy (SEM) images were collected on an S-4800 field emission SEM (FESEM, Hitachi, Tokyo, Japan). Transmission electron microscopy (TEM) and high-resolution transmission electron microscopy (HRTEM) analyses were conducted with a JEM-2100F electron microscope (JEOL, Tokyo, Japan). Thermal gravimetric analysis (TGA, STA449f3, Netzsch, Selb, Germany) was performed in air at a heating rate of $10^{\circ} \mathrm{C} \cdot \mathrm{min}^{-1}$. The Fourier transform infrared spectra (FT-IR) of the samples were recorded on a TENSOR27 FT-IR spectrometer (Bruker, Berlin, Germany). C solid-state magic angle spinning (MAS) NMR experiments were acquired on Bruker Avance $300 \mathrm{MHz}$ (7 T) spectrometer using the $4 \mathrm{~mm}$ zirconia rotors as sample holders spinning at MAS rate $v_{\text {MAS }}=14 \mathrm{kHz}$. The Brunauer-Emmett-Teller $(\mathrm{BET})$ surface area was determined by a multipoint BET method. The UV-vis diffuse reflectance spectra $(220-800 \mathrm{~nm})$ were recorded using a 
UV-vis spectrophotometer (UV2550, Shimadzu, Kyoto, Japan). Photoluminescence (PL) spectra were measured using a fluorescence spectrophotometer (Fluorolog-3, HORIBA Scientific, Edison, NJ, USA) with an excitation wavelength of $315 \mathrm{~nm}$.

\subsection{Photocatalytic Degradation of RhB Measurements}

For a typical test, $20 \mathrm{mg}$ of as-prepared photocatalyst was added into $20 \mathrm{~mL}$ of $10 \mathrm{ppm}$ of RhB solution. After adsorption-desorption equilibrium in the dark for 30 minutes, the solution was illuminated by a $300 \mathrm{~W}$ xenon lamp with a $420 \mathrm{~nm}$ cutoff filter providing visible light irradiation. The light intensity was measured to be about $120 \mathrm{~mW} / \mathrm{cm}^{2}$. During the photocatalytic process, $4 \mathrm{~mL}$ of the reaction solution was centrifuged to separate the photocatalyst and $\mathrm{RhB}$ solution after irradiation for a certain period, and then tested using UV-vis spectrometer.

\section{Conclusions}

In summary, we have developed a facile one-pot hydrothermal preparation method to synthesize HTCC-coated CdS nanocomposite assisted by glucose and polyvinylpyrrolidone (PVP). The as-prepared HTCC-coated CdS showed superior photocatalytic activity for the degradation of $\mathrm{RhB}$ under visible light irradiation $(\lambda \geq 420 \mathrm{~nm}$ ). The sample (glucose content of $0.1 \mathrm{~g}$ ) showed a degradation rate four-times that of pure CdS reference. Moreover, it also showed an improved stability, and the activity could maintain at $96.2 \%$ after three cycles of recycling. The enhanced photocatalytic activity and stability of this nanocomposite can be mainly attributed to: (i) The addition of PVP in the reaction solution can significantly increase the specific surface area of $\mathrm{CdS}$, and thus offer more active sites; (ii) The HTCC in the nanocomposite can expand the range of light absorption; (iii) The HTCC layer can form a heterojunction with $\mathrm{CdS}$ and improve the charge separation and transfer. This novel CdS-based nanocomposite exhibits great potential in environmental remediation.

Supplementary Materials: The following are available online at http://www.mdpi.com/2073-4344/7/7/194/s1. Figure S1: ${ }^{13} \mathrm{C}$ Solid-state NMR of the as-prepared HTCC, the chemicals shift peaks of polyfuran domains are highlighted here; Figure S2: HRTEM images of (a) 0.05C/CdS-PVP and (b) 0.5C/CdS-PVP samples; Figure S3: TGA curves of CdS-PVP and 0.1C/CdS-PVP samples; Figure S4: The pore size distribution curves of the pure CdS, CdS-PVP and 0.1C/CdS-PVP samples; Figure S5: The band gaps calculated from Kubelka-Munk plots of the as-prepared samples; Figure S6: SEM images of (a) 0.2C/CdS-PVP and (b) 0.5C/CdS-PVP samples.

Acknowledgments: This work was supported by the National Natural Science Foundation of China (No.21303118), the Doctor Project for Young Teachers of Ministry of Education (No.20130032120003), the Seed Foundation of Tianjin University (No.1501) and the research program of application foundation of Qinghai province (2017-zj-729).

Author Contributions: Yuanliang Ma and Qiang Cai and Zhurui Shen conceived and designed the experiments; Yuanliang Ma performed the experiments; Yuanliang Ma, Qiang Cai and Zhurui Shen analyzed the data. Zhongkun Zhao, Huiming Ji and Leichao Meng contributed reagents and analysis tools. Yuanliang Ma and Qiang Cai wrote the paper.

Conflicts of Interest: The authors declare no conflict of interest.

\section{References}

1. Chen, X.B.; Shen, S.H.; Guo, L.J.; Mao, S.S. Semiconductor-based photocatalytic hydrogen generation. Chem. Rev. 2010, 110, 6503-6570. [CrossRef] [PubMed]

2. Chong, M.N.; Jin, B.; Chow, C.W.K.; Saint, C. Recent developments in photocatalytic water treatment technology: A review. Water Res. 2010, 44, 2997-3027. [CrossRef] [PubMed]

3. Fujishima A, H.K. Electrochemical photolysis of water at a semiconductor electrode. Nature 1972, 238, 37-38. [CrossRef]

4. Li, Y.; Ouyang, S.; Xu, H.; Wang, X.; Bi, Y.; Zhang, Y.; Ye, J. Constructing solid-gas-interfacial fenton reaction over Alkalinized- $\mathrm{C}_{3} \mathrm{~N}_{4}$ photocatalyst to achieve apparent quantum yield of $49 \%$ at $420 \mathrm{~nm}$. J. Am. Chem. Soc. 2016, 138, 13289-13297. [CrossRef] [PubMed] 
5. Kim, T.W.; Choi, K.S. Nanoporous $\mathrm{BiVO}_{4}$ photoanodes with dual-layer oxygen evolution catalysts for solar water splitting. Science 2014, 343, 990-994. [CrossRef] [PubMed]

6. Hu, Z.; Yuan, L.; Liu, Z.; Shen, Z.; Yu, J.C. An elemental phosphorus photocatalyst with a record high hydrogen evolution efficiency. Angew. Chem. 2016, 128, 9732-9737. [CrossRef]

7. Xiang, Q.; Cheng, B.; Yu, J. Hierarchical porous CdS nanosheet-assembled flowers with enhanced visible-light photocatalytic H-2-production performance. Appl. Catal. B Environ. 2013, 138, 299-303. [CrossRef]

8. Jin, J.; Yu, J.; Liu, G.; Wong, P.K. Single crystal CdS nanowires with high visible-light photocatalytic H-2-production performance. J. Mater. Chem. A 2013, 1, 10927-10934. [CrossRef]

9. Hu, Y.; Gao, X.H.; Yu, L.; Wang, Y.R.; Ning, J.Q.; Xu, S.J.; Lou, X.W. Carbon-coated CdS petalous nanostructures with enhanced photostability and photocatalytic activity. Angew. Chem. Int. Ed. 2013, 52, 5636-5639. [CrossRef] [PubMed]

10. Chen, J.; Wu, X.J.; Yin, L.; Li, B.; Hong, X.; Fan, Z.; Chen, B.; Xue, C.; Zhang, H. One-pot synthesis of CdS nanocrystals hybridized with single-layer transition-metal dichalcogenide nanosheets for efficient photocatalytic hydrogen evolution. Angew. Chem. Int. Ed. 2015, 54, 1210-1214. [CrossRef] [PubMed]

11. Li, Q.; Li, X.; Wageh, S.; Al-Ghamdi, A.A.; Yu, J. CdS/Graphene nanocomposite photocatalysts. Adv. Energy Mater. 2015, 5. [CrossRef]

12. Xie, Y.P.; Yu, Z.B.; Liu, G.; Ma, X.L.; Cheng, H.M. CdS-mesoporous ZnS core-shell particles for efficient and stable photocatalytic hydrogen evolution under visible light. Energy Environ. Sci. 2014, 7, 1895-1901. [CrossRef]

13. Wang, X.; Liu, G.; Wang, L.; Chen, Z.G.; Lu, G.Q.; Cheng, H.-M. ZnO-CdS@Cd heterostructure for effective photocatalytic hydrogen generation. Adv. Energy Mater. 2012, 2, 42-46. [CrossRef]

14. Li, Q.; Guo, B.; Yu, J.; Ran, J.; Zhang, B.; Yan, H.; Gong, J.R. Highly efficient visible-light-driven photocatalytic hydrogen production of CdS-cluster-decorated graphene nanosheets. J. Am. Chem. Soc. 2011, 133, 10878-10884. [CrossRef] [PubMed]

15. Neelgund, G.M.; Oki, A. Photocatalytic activity of CdS and $\mathrm{Ag}_{2} \mathrm{~S}$ quantum dots deposited on poly(amidoamine) functionalized carbon nanotubes. Appl. Catal. B Environ. 2011, 110, 99-107. [CrossRef] [PubMed]

16. Chen, J.; Zhang, F.; Zhao, Y.L.; Guo, Y.C.; Gong, P.; Li, Z.Q.; Qian, H.S. Facile synthesis of CdS/C core-shell nanospheres with ultrathin carbon layer for enhanced photocatalytic properties and stability. Appl. Surf. Sci. 2016, 362, 126-131. [CrossRef]

17. Cai, Q.; Hu, Z.; Zhang, Q.; Li, B.; Shen, Z. Fullerene (C60)/CdS nanocomposite with enhanced photocatalytic activity and stability. Appl. Surf. Sci. 2017, 403, 151-158. [CrossRef]

18. Falco, C.; Baccile, N.; Titirici, M.M. Morphological and structural differences between glucose, cellulose and lignocellulosic biomass derived hydrothermal carbons. Green Chem. 2011, 13, 3273-3281. [CrossRef]

19. Hu, Z.; Liu, G.; Chen, X.; Shen, Z.; Yu, J.C. Enhancing charge separation in metallic photocatalysts: A case study of the conducting molybdenum dioxide. Adv. Funct. Mat. 2016, 26, 4445-4455. [CrossRef]

20. Heiba, Z.K.; Mohamed, M.B.; Imam, N.G. Structural tuning of CdS nanoparticles with nucleation temperature and its reflection on the optical properties. J. Mol. Struct. 2015, 1094, 91-97. [CrossRef]

21. Ge, L.; Zuo, F.; Liu, J.; Ma, Q.; Wang, C.; Sun, D.; Bartels, L.; Feng, P. Synthesis and efficient visible light photocatalytic hydrogen evolution of polymeric $\mathrm{g}-\mathrm{C}_{3} \mathrm{~N}_{4}$ coupled with cds quantum dots. J. Phys. Chem. C 2012, 116, 13708-13714. [CrossRef]

22. Glenis S, B.M.; LeGoff, E. Polyfuran-A new synthetic approach and electronic-properties. J. Am. Chem. Soc. 1993, 115, 12519-12525. [CrossRef]

23. Hao, R.; Xiao, X.; Zuo, X.; Nan, J.; Zhang, W. Efficient adsorption and visible-light photocatalytic degradation of tetracycline hydrochloride using mesoporous BiOI microspheres. J. Hazard Mat. 2012, 209, 137-145. [CrossRef] [PubMed] 
24. Shi, X.; Chen, X.; Chen, X.; Zhou, S.; Lou, S.; Wang, Y.; Yuan, L. PVP assisted hydrothermal synthesis of BiOBr hierarchical nanostructures and high photocatalytic capacity. Chem. Eng. J. 2013, 222, 120-127. [CrossRef]

25. Hu, Z.; Shen, Z.; Yu, J.C.M. Converting carbohydrates to carbon-based photocatalysts for environmental treatment. Environ. Sci. Technol. 2017. [CrossRef] [PubMed] 